\title{
OBSERVATIONS ON THE PROPHYLAXIS OF EXPERIMENTAL PULMONARY ANTHRAX IN THE MONKEY
}

\author{
By D. W. HENDERSON, S. PEACOCK and F. C. BELTON \\ Microbiological Research Department, Porton, near Salisbury, Wilts.
}

(With 4 Figures in the Text)

\section{INTRODUCTION}

At the time this work commenced there was evidence to suggest that experimental inhalation anthrax in the guinea-pig was not a true pulmonary disease (Barnes, 1947); it was probably an example of the introduction of virulent organisms into the body through the lymphatic system of undamaged lung with the subsequent development of characteristic general infection. But technical difficulties had prevented direct observation of the behaviour of the inhaled spores on lung epithelium. The inference that the disease developed in this way followed from the observation that organisms were first seen multiplying in the tracheobronchial lymph glands. However, there was some supporting but indirect evidence which showed that undamaged and heat resistant spores could be recovered from guinea-pig lung for at least $24 \mathrm{hr}$. after their deposition. This suggested that lung epithelium might not be a suitable place for spore germination and multiplication. While the present work was in progress Dr Ross, in this Department, re-investigated the fate of anthrax spores deposited on lung epithelium of the guinea-pig. She extended Barnes's (1947) findings by developing a histological technique which allowed direct observation of deposited spores and their subsequent behaviour. By this means confirmation was obtained that the site of initiation of infection in this experimental form of pulmonary anthrax is lymphatic tissue (Ross, 1956).

The experiments now described had two objectives. It was of interest to find if transitory prophylaxis (e.g. with penicillin) would be effective against this form of disease. If the hypothesis on pathogenesis was correct, then penicillin might be effective only so long as it was continuously present and until the spores had been completely removed from the lung. If, on the other hand, the organisms obtained access to lung tissue, the antibiotic might be speedily effective in ridding the host of potential infection. In the event that the former finding held, the second objective was to attempt to develop alternative prophylactic measures that could be commenced after exposure of the host to infection by the pulmonary route.

\section{METHODS}

Infective agent. A highly virulent strain of Bacillus anthracis was used. Its origin and infectivity, and the method of production of the spore suspensions used for cloud production, have been described (Druett, Henderson, Packman \& Peacock, 1953).

Animals. Macacus rhesus monkeys weighing 7-14 lb. were used. They were 
housed singly in cages approximately $3 \times 2 \times 1 \frac{1}{2} \mathrm{ft}$. Confirmation of death from anthrax was obtained by microscopic and cultural examination of the heart's blood.

Apparatus for exposing animals to infection. Clouds of single spores were produced in the apparatus previously described (Henderson, 1952).

Cloud sampling and method of expressing dosage. As previously described (Druett et al. 1953).

Prophylactic measures. (a) Administration of procaine penicillin. (b) Administration of immune serum. (c) Penicillin prophylaxis combined with active immunization.

Details of procedure are given in the description of experiments.

Spore content of lungs. Where an estimate of spore numbers was to be made, animals were sacrificed by the intraperitoneal injection of $10 \mathrm{ml}$. of Nembutal. Immediately after death the thorax was exposed and main pulmonary vessels clamped with forceps. Lungs were removed by severing the trachea immediately above the bifurcation of the bronchus. The right and left lungs were dissected apart and placed in previously weighed beakers. The weight of lung was noted and the beakers placed immediately in an ice-salt mixture. Approximately $\mathbf{l ~ h r}$. later the tissues were chopped with scissors and placed in previously chilled mortars $\left(0-4^{\circ} \mathrm{C}\right.$.). Two $\mathrm{ml}$. of saline per $\mathrm{g}$. of tissue were slowly added while the tissue was ground by pestle. These latter operations were carried out at $0-4^{\circ} \mathrm{C}$. Two portions of the resulting extract were tested for viable anthrax organisms; one was plated immediately to give a total count, the other heated at $60^{\circ} \mathrm{C}$. for $1 \frac{1}{2} \mathrm{hr}$. to give a count of remaining heat-resistant spores. Tryptic meat agar was the substrate for growth, and counts were made by spreading $0.2 \mathrm{ml}$. amounts of the extract or dilution thereof over the surface of the containing Petri dish.

\section{RESULTS}

Penicillin as a prophylactic. In an early experiment twenty monkeys, varying in weight from $7 \frac{1}{2}$ to $11 \frac{1}{2} \mathrm{lb}$., were exposed to an LNt of 0.214 . This is equivalent to a dosage of about $4 \mathrm{LNt} 50$ and represents an inhaled dose of about $2 \cdot 14 \times 10^{5}$ spores. Twenty-four hours after exposure ten animals received 150,000 units $(0.5 \mathrm{ml}$.) of procaine penicillin intramuscularly in the right thigh. The remainder, acting as controls, received similarly $0.5 \mathrm{ml}$. of physiological saline. This process was repeated four times at $24 \mathrm{hr}$. intervals, i.e. in all, 750,000 units of penicillin were given over a period of 5 days. Results are shown graphically in Fig. 1. Death among control monkeys commenced on the 5th day after exposure. Eight of ten were dead by the 10th day and a 9th animal died of anthrax on the 23rd day. One remained alive until the experiment was terminated on the 49th day. The first death from anthrax in the treated animals was delayed until the 14th day after exposure (i.e. 9 days after discontinuing penicillin treatment). Thereafter, the course of events followed closely the pattern of the controls, and eventually by the 33rd day eight of ten treated animals were dead from anthrax (Fig. 1).

This finding clearly indicated that penicillin treatment prevented the onset of 
anthrax, and suggested that if the penicillin level had been maintained for an indefinite period the animals would have been saved. It also indicated the possibility already outlined that the anthrax spores deposited on lung epithelium sat as inert particles awaiting the chance of being picked up by a wandering macrophage and transported to a lymph gland where germination and multiplication could take place.

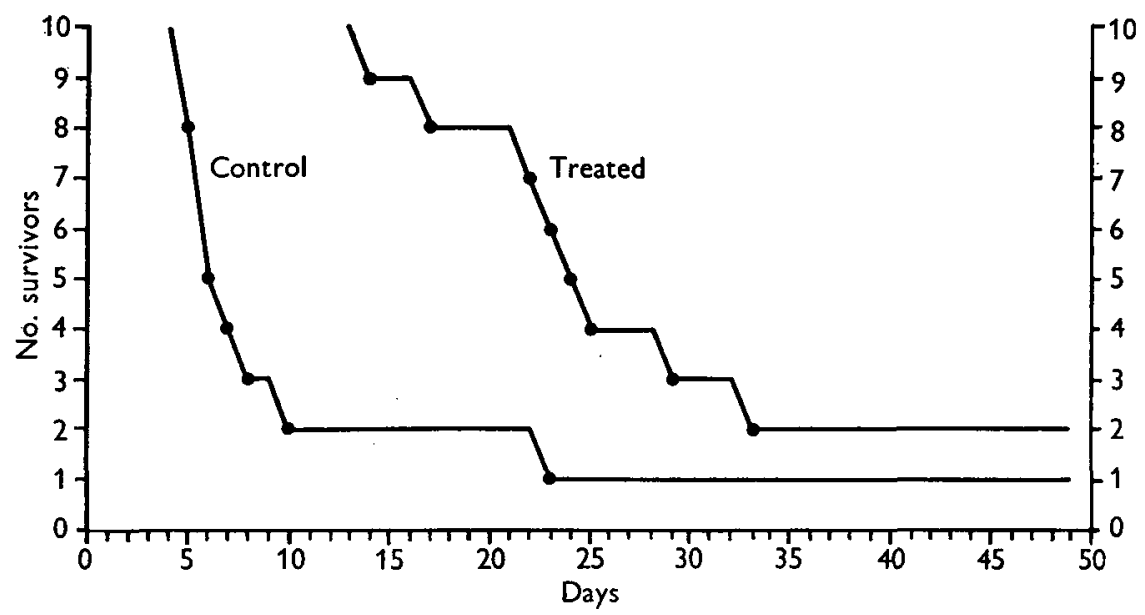

Fig. 1. Penicillin treatment of pulmonary anthrax in monkeys. Inhalation dose: $2 \times 10^{5}$ spores/litre cloud $=4 \mathrm{LNt}$ 50. Treatment: $1.5 \times 10^{5}$ units i.m. $24 \mathrm{hr}$. after exposure. Similar daily treatment for a further 4 days.

In another experiment fifty-four animals were used, varying in weight from $7 \frac{1}{4}$ to $10 \mathrm{lb}$. They were exposed to an LNt of $0 \cdot 385$. This represents a dosage of about $8 \mathrm{LNt50}$, and an estimated intake per animal of about $3.9 \times 10^{5}$ spores. Forty animals were selected at random for the main experiment and placed in four groups of ten. (The remainder, as described later, were killed at various intervals to examine the lungs for spore content.) One group served as control and was treated as in the first experiment. The second, group A, received penicillin as in the first experiment, i.e. five daily doses each of 150,000 units commencing $24 \mathrm{hr}$. after exposure. The third, group B, was similarly treated but with extension of the penicillin administration daily for 10 days. The fourth group, group C, received in the same manner penicillin over a period of 20 days. The results are shown graphically in Fig. 2. First, it is seen that the results with control animals and those receiving penicillin for 5 days form an almost exact replica, in time to death and in numbers dying, of the picture in the first experiment. The minor exceptions are that here the 10th control animal died of anthrax on the 8th day and the 10th animal in the treated group died non-specifically on the 59th day after exposure; post mortem examination showed that it suffered from fairly extensive pulmonary tuberculosis. The pattern obtained in group B, treated for 10 days, is simply a further delayed form of that obtaining in the control group. It is of interest here that the 10th animal to die of anthrax did not do so until the 42nd day after exposure; it had pulmonary tuberculosis. The fourth group, 
group C, treated for 20 days was ill-fated for reasons other than exposure to anthrax. Maybe the continuous handling, let alone the heavy penicillin treatment, was responsible. One animal died non-specifically on the 10th day; Shigella dysenteriae (Schmitzii) was isolated from the gut content. Similarly, on the 14th, 18th and 29th days respectively an animal was found dead from a disease other than anthrax. This depleted group was retained until the 42nd day after exposure when the remaining animals were sacrificed for examination of spore content of the lung. In the interval, on the 36th and 38th days respectively two animals died of anthrax. This is a slender picture of likely events, but it is included as an indication of the probable outcome in an otherwise normal experiment. The line shown in Fig. 2, representing this group, is imaginative in the sense that only two specific anthrax deaths in the remaining animals fixed the slope of the likely event.

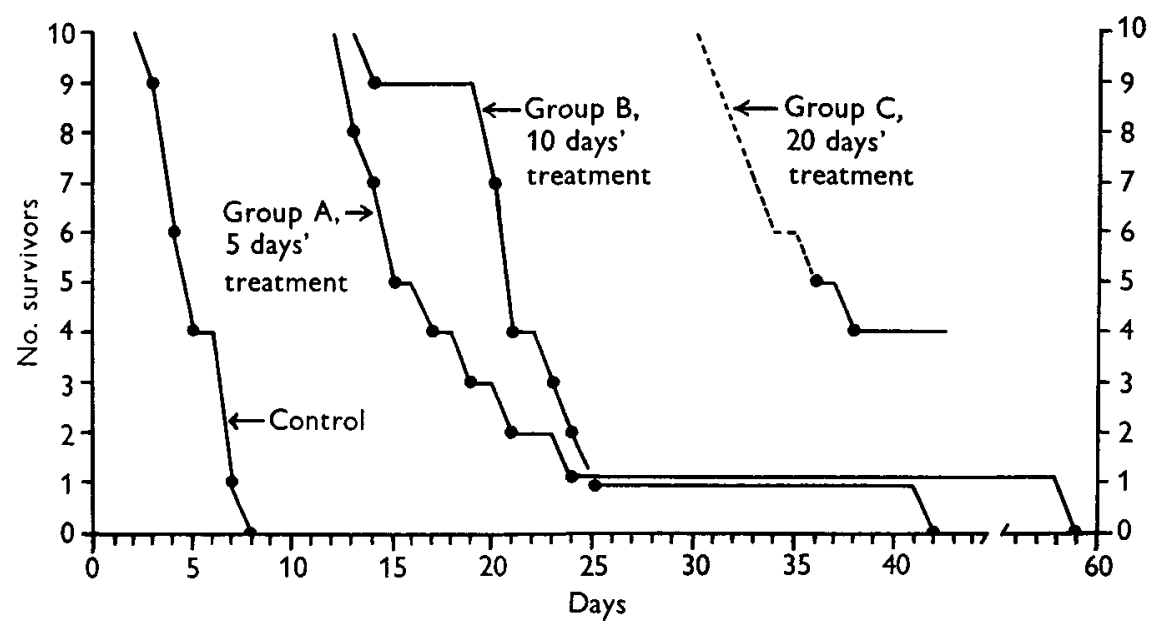

Fig. 2. Effect of 5, 10 and 20 days' penicillin treatment of pulmonary anthrax in monkeys. Inhalation dose : $4 \times 10^{5}$ spores/litre cloud $=8$ LNt 50. Treatment: $1.5 \times 10^{5}$ units i.m. $24 \mathrm{hr}$. after exposure. Similar daily treatment for a further 4 days for group $A, 9$ days for group $B$ and 19 days for group $C$.

Immune serum as a prophylactic. Hyper-immune serum was prepared in the horse by repeated injection of avirulent spore vaccine prepared according to the method described by Sterne (1937). This serum completely protected rabbits when given intravenously in $1 \mathrm{ml}$. amounts $24 \mathrm{hr}$. before an intracutaneous injection of anthrax spores containing about 250 ALD. Experiments were planned using the serum on similar lines to those adopted in the tests with penicillin. The details of one trial are as follows. Thirty monkeys were used, ranging in weight from $4 \frac{1}{2}$ to $6 \mathrm{lb}$. (this discrepancy in weight from the other experiments was dictated solely by the availability of animals). All monkeys were exposed to an inhalation dose of about $2 \times 10^{5}$ spores representing approximately 4 LNt50. A group of ten served as controls; each animal received two intramuscular injections in the thigh of $6 \mathrm{ml}$. of normal horse serum on the 1st and 6th day respectively after exposure. A second group of ten received similarly two intra- 
muscular injections of $6 \mathrm{ml}$. of a concentrate (fivefold) of the immune serum described above. A third group received one intramuscular injection of $6 \mathrm{ml}$. of immune serum on the first day after exposure. The results are shown graphically in Fig. 3. They show the same general pattern as in the experiments on penicillin prophylaxis. No deaths occurred in the group given one dose of immune serum until 20 days after exposure, and not until the 27th day in the group given two doses. On the other hand, seven of ten controls were dead of anthrax by the 8th day, and nine eventually died. However, by the time the experiment was termi-

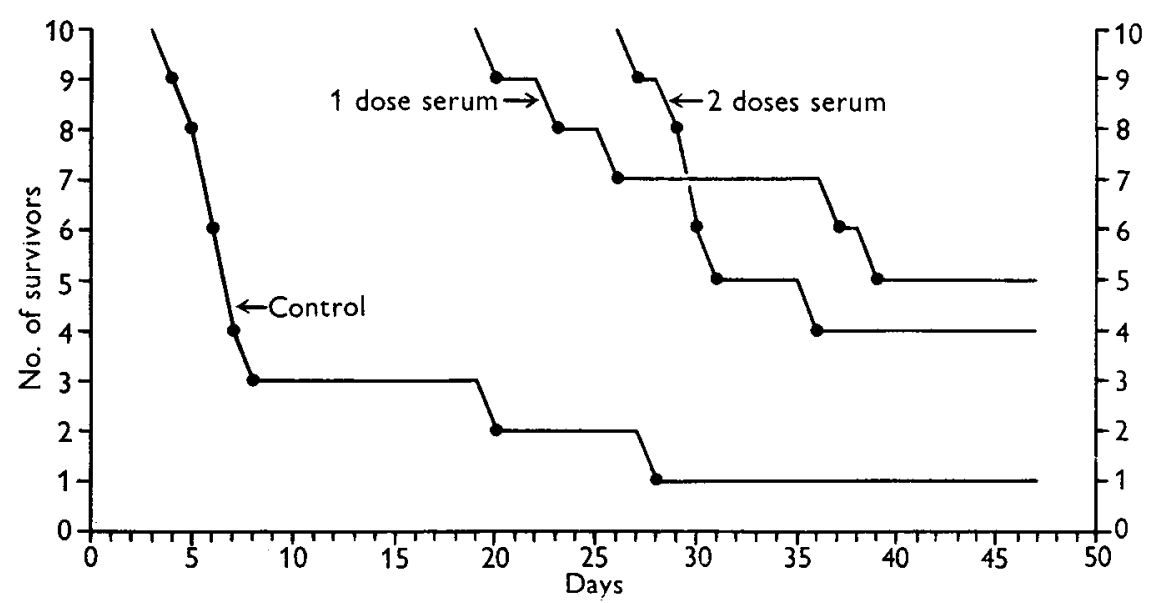

Fig. 3. Serum treatment of pulmonary anthrax in monkeys. Inhalation dose : $2 \times 10^{5}$ spores/ litre cloud $=4 \mathrm{LNt} 50$. Treatment: one group given 1 dose serum i.m. $24 \mathrm{hr}$. after exposure; second group similarly treated and then a further dose on the 5th day.

nated on the 47th day after exposure only $45 \%$ of the immune serum-treated animals survived. Reference will be made later to this point, when the time of lung retention of anthrax spores is examined.

\section{Penicillin prophylaxis combined with active immunization}

Wright, Hedberg \& Slein (1954) and Belton \& Strange (1954) have shown that a highly effective and wholly innocuous soluble protective antigen can be prepared by suitable in vitro culture of $B$. anthracis. Belton \& Strange showed that two small doses of this antigen (alum precipitated), given to monkeys with a 10-day interval between doses, produced a solid immunity to a respiratory challenge of anthrax spores presented 7 days after the second dose of vaccine. This led to exploration of the possibility of combined penicillin and vaccine prophylaxis commencing after exposure to anthrax spores.

An experiment was made with forty monkeys. Ten (group $A$ ) acted as nonimmune controls; ten (group $B$ ) were vaccinated, prior to exposure, after the method of Belton \& Strange; ten (group $C$ ) received a total of 750,000 units of penicillin given in 5 doses at daily intervals commencing $24 \mathrm{hr}$. after exposure; ten (group $D$ ) were similarly treated with penicillin but received, in addition, two doses of vaccine as in the vaccinated control group, the first dose $24 \mathrm{hr}$. after 
exposure, the second 10 days later. All animals were exposed to approximately 15 LNt50 doses of anthrax spores. The results, shown graphically in Fig. 4, are self-explanatory. The penicillin picture is strongly reminiscent of that shown in Fig. 1. Previous vaccination protected all animals, and the combined vaccine and penicillin procedure had a like effect. The penicillin 'cover' thus given is sufficient to allow effective immunity to be developed by vaccination commencing after exposure to infection. The experiment was terminated on the 50th day after exposure. However, there is evidence to show that monkeys actively immunized retain effective resistance against respiratory challenge for at least a year and perhaps much longer (Belton, Henderson \& Peacock, 1956).

Table 1. Assay of Bacillus anthracis in monkey lung after inhalation of spores

(Estimated retained dose $1 \times 10^{5}$ spores.)

$\begin{array}{cccc}\begin{array}{c}\text { Days after } \\ \text { exposure }\end{array} & \begin{array}{c}\text { Monkey } \\ \text { no. }\end{array} & \text { Unheated } & \text { Heated } \\ 3 & 5 & 10 & 7 \\ & 40 & 4 & 4 \\ 5 & 11 & 5 & 4 \\ & 20 & 9 & 6 \\ & 41 & 2 & 0 \cdot 9 \\ 7 & 48 & 3 & 1 \\ & 18 & 6 & 4 \\ 10 & 49 & 5 & 3 \\ & 4 & 9 & 5 \\ & 19 & 9 & 4 \\ 15 & 30 & 4 & 3 \\ & 43 & 7 & 1 \\ & 31 & 9 & 5 \\ \text { Average 3-15 days } & 54 & 9 & 5 \\ 42 & & 6 \cdot 5 & 4 \cdot 4 \\ & 23 & 1 & 0 \cdot 9 \\ & 28 & 2 & 2 \\ & (47) & (8) & (5) \\ & 50 & 2 & 2\end{array}$

Figures given to the nearest decimal place.

Survival of anthrax spores in monkey lung. Harper \& Morton (1953) showed that monkeys were highly variable in respect of retention of inhaled particles and their site of deposition. This is due in large measure to the behaviour of monkeys under experiment; rate and depth of breathing, holding of breath for short periods, and mouth breathing are the main contributory factors. Their data showed that the total retention of single spores was about $25 \%$ of that inhaled, and of this number about $60 \%$ were to be found in the lung. In one of the present experiments (Fig. 2), to determine the usefulness of penicillin as a prophylactic, extra animals were included. They were sacrificed at various intervals after exposure to determine the number of spores (resistant and non-resistant to heat) that could be detected in the lungs. The results are given in Table 1. The estimated retained dose 
in the lung was $1 \times 10^{5}$ spores. It is seen that up to 42 days after exposure, large numbers of spores were recoverable, but there was no evidence of increase in numbers of $B$. anthracis at any time. The results follow the irregular pattern found by Harper \& Morton, but when averaged it is seen that at any time up to 15 days after exposure about $65 \%$ of the originally retained spores are recoverable, but some $30 \%$ of these are heat-sensitive. By the 42nd day (in monkeys that had received penicillin for 20 days after exposure), with one exception, the percentage of originally retained organisms recoverable was markedly reduced to about $15-20 \%$ of the originally retained spores.

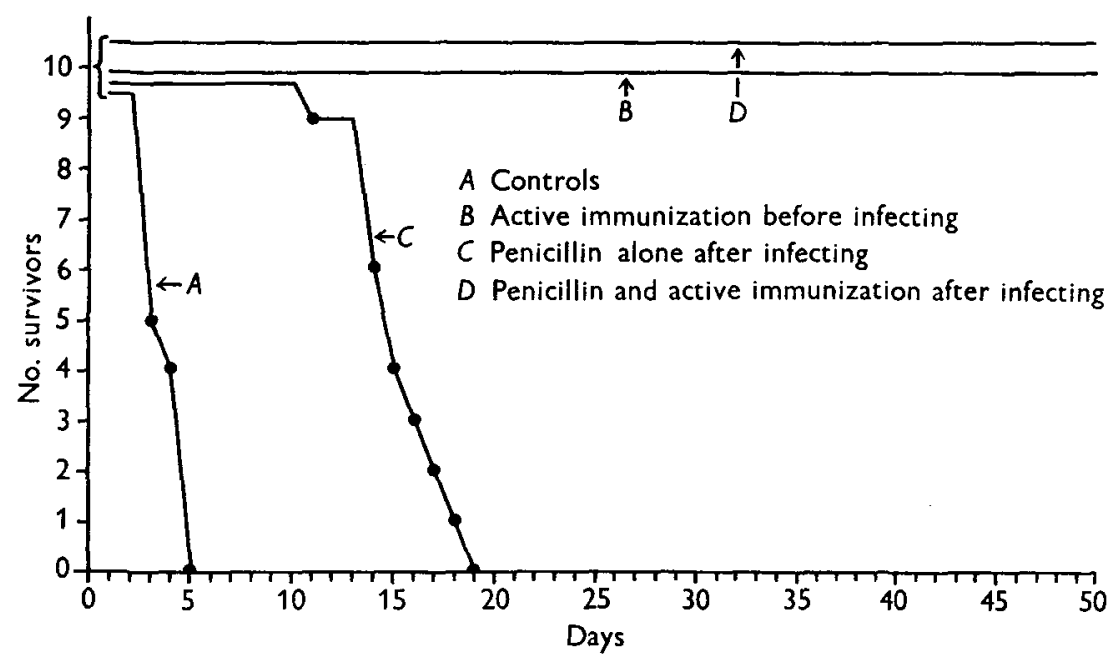

Fig. 4. Combined penicillin and active immunization of monkeys. Inhalation dose: $7.59 \times 10^{5}$ spores/litre cloud $=15 \mathrm{LNt}$ 50. Penicillin treatment: $1.5 \times 10^{5}$ units i.m. $24 \mathrm{hr}$. after exposure. Similar treatment daily for a further 4 days. Active immunization: first dose of $1.0 \mathrm{ml}$. of $\times 5$ filtrate subcut. $24 \mathrm{hr}$. after exposure. Second dose 10 days later.

Data on longer period survival on lung were obtained by examining vaccinated animals surviving after exposure to single spore clouds. For instance, survivors from the experiment on combined penicillin and vaccine prophylaxis, described above (Fig. 4), were examined at 50 days (five animals), 75 days (five animals) and 100 days (nine animals) after exposure. Detailed figures for recovery have little quantitative significance in this experiment, for the number of colonies developing per Petri dish ranges from 0 to 20 . However, combined data from all tests, which involved examination of some fifty animals, show the trend of events as follows:

Time after exposure (days)

42

50

75

100
Estimated percentage of original retention

$15-20$

2

$0 \cdot 5-1$

Traces 


\section{DISCUSSION}

The experiments have confirmed the hypothesis based on earlier findings relative to the pathogenesis of inhalation anthrax (Barnes, 1947) that transitory methods of prophylaxis would be ineffective. Examination of the fate of spores deposited on lung epithelium has offered an explanation for the findings. It appears that only a very small proportion of the deposited spores find their way, or are transported, to the lymph glands to be dealt with by whichever prophylactic measure is adopted. The great majority remain for long periods unaffected; their presence can be detected for at least 100 days after deposition in the lung. Further, the technique for their detection was limited in efficiency because of the quantity of tissue that could be examined, so the period of survival recorded is likely to be minimal. In addition, it was found that a high percentage of recovered spores were heat resistant. And again it must be understood that the technique for lung examination had limitations. Unless very special precautions were taken to chill tissue and utensils to $0-4^{\circ}$ before grinding, the contained spores very rapidly entered the first stage of germination, rendering them heat-sensitive. The figures quoted in the text, therefore, for heat-resistant forms are also minimal. However, from various control experiments to check the technique we are satisfied that at least a very high proportion of the heat-sensitive spores detected were in this state in vivo. It seems probable that in some slow but eventually effective manner a proportion of retained spores emerge from their shell but fail to multiply and eventually die. Weight is given to this reasoning by the fact that no animals showed any increased numbers of organisms over those estimated to have been originally retained in the lung. The possibility that the immune or otherwise protected animals prevented multiplication of germinating spores on lung epithelium may be excluded. It was found that animals given penicillin but not examined until weeks after the last injection of the antibiotic showed no increase in total bacterial population. Further, the proportion of heat-sensitive to heat-resistant forms remained of the same order at all times after exposure. A process of slow self-extinction of spores seems operative in ridding the lung of most retained spores but, until these have been disposed of, occasional spores may reach the lymphatics, in which situation the host can deal with them only if in a continuous state of prophylactic readiness.

\section{SUMMARY}

The evidence presented supports the hypothesis that the experimental form of anthrax induced by depositing a cloud of single spores on lung epithelium is initiated in the lymphatic regions and not in lung tissue.

Proportionately few of the deposited spores reach the lymph glands. The majority remain inactive in so far as induction of disease is concerned. Slowly, however, they seem to go through the first stages of germination but do not multiply and therefore die. This is probably the major factor operative in their disappearance from the lung.

Effective prophylactic measures are suggested for dealing with an unimmunized host exposed to anthrax infection by the pulmonary route. 


\section{REFERENCES}

Barnes, J. M. (1947). Brit. J. exp. Path. 28, 385.

Belton, F. C. \& Strange, R. E. (1954). Brit. J. exp. Path. 35, 144.

Belton, F. C., Henderson, D. W. \& Peacock, S. (1956). Brit. J. exp. Path. (in the Press).

Druetw, H. A., Henderson, D. W., Packman, L. \& Peacock, S. (1953). J. Hyg., Camb., 51, 359.

Harper, G. J. \& Morton, J. D. (1953). J. Hyg., Camb., 51, 372.

Henderson, D. W. (1952). J. Hyg., Camb., 50, 53.

Ross, J. (1956). J. Path. Bact. (in the Press).

STERNE, M. (1937). Ondestepoort J. vet. Sci. 8, 271.

Wright, G. G., Hedberg, Mary A. \& Slein, J. B. (1954). J. Immunol. 72, 263.

(MS. received for publication 25. vII. 55) 